\title{
Effect of Molecular Architecture on the Self-Assembly of Bottlebrush Copolymers
}

Karim R. Gadelrab and Alfredo Alexander-Katz

Department of Materials Science and Engineering. Massachusetts Institute of Technology, Cambridge, Massachusetts, 02139, United States.
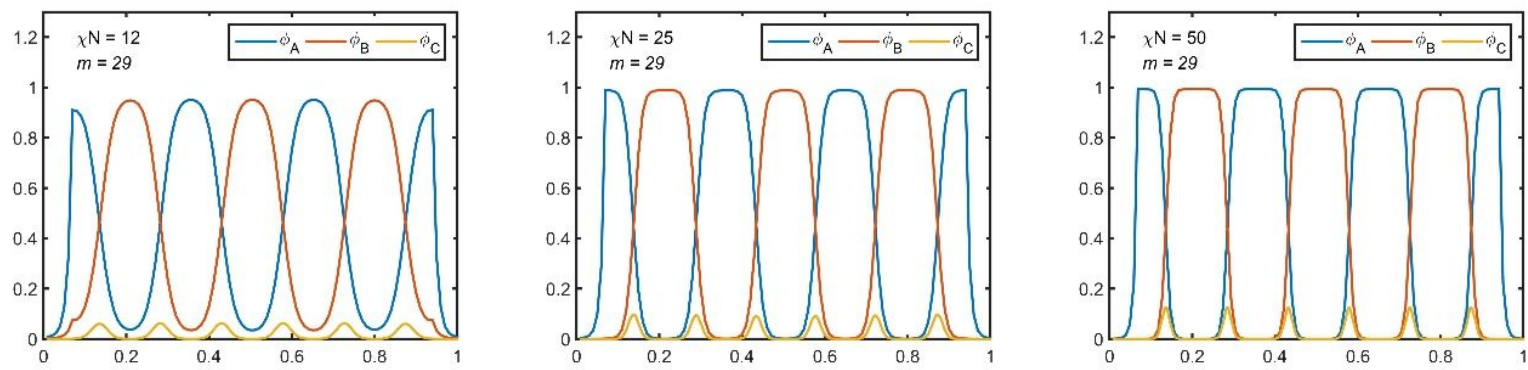

Figure S1. Density profiles for polymer domains lying parallel to the confining walls. Density vanishes at extremities due to the applied external fields. Increasing $\chi N$ resulted in a stronger AB segregation with sharper interface. The incompressibility constraint localized the neutral block $C$ at the $A B$ interface.
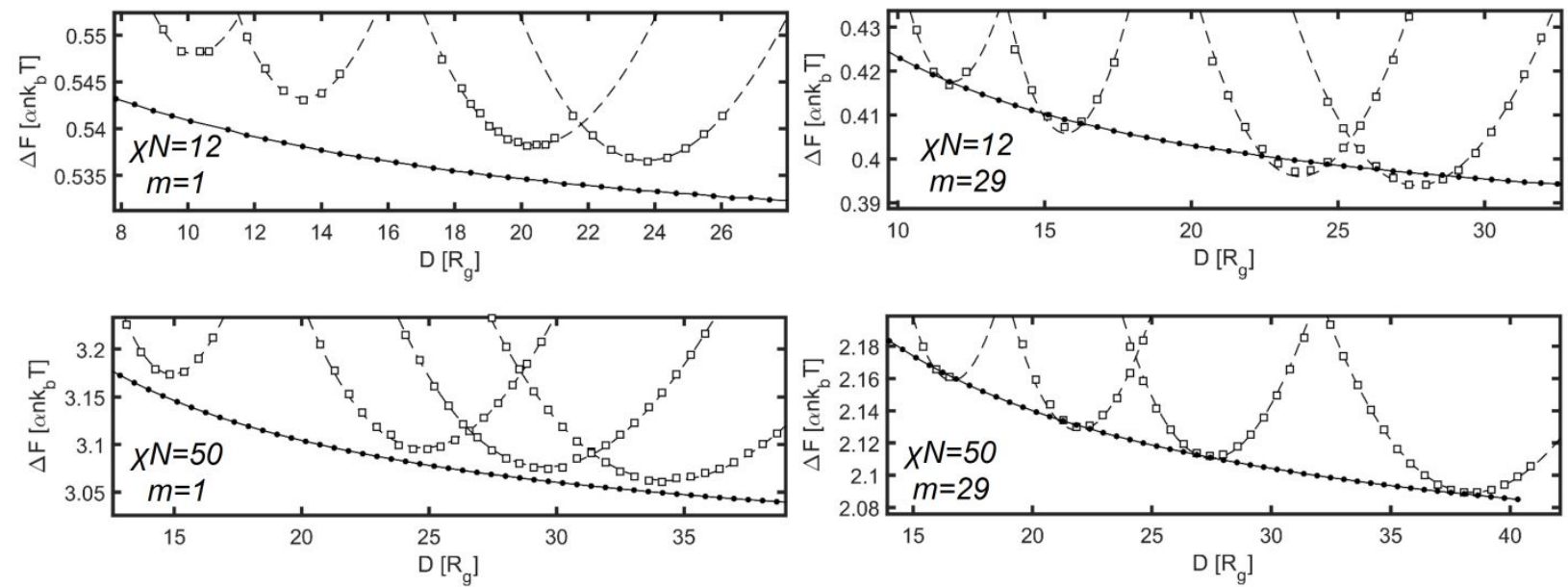

Figure $S 2$. Free energy $\Delta F$ plots as function of trench width $D\left(3 L_{0}\right.$ to $\left.7 L_{0}\right)$ for varying segregation strength $\chi N$ and number of AB sidechains $m$. 


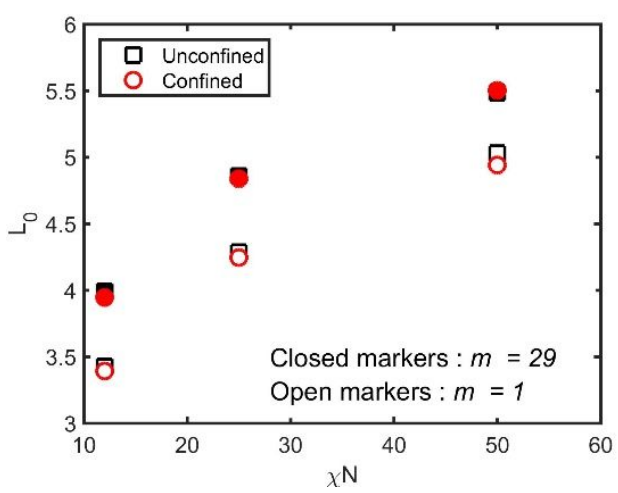

Figure S3. Equilibrium domain spacing $L_{0}$ for different simulation conditions extracted from confined simulations (circular markers) compared to the corresponding bulk values (square markers). Bottlebrush polymers with $m=29$ have consistently larger $L_{0}$ indicating extended $A B$ sidechains. 

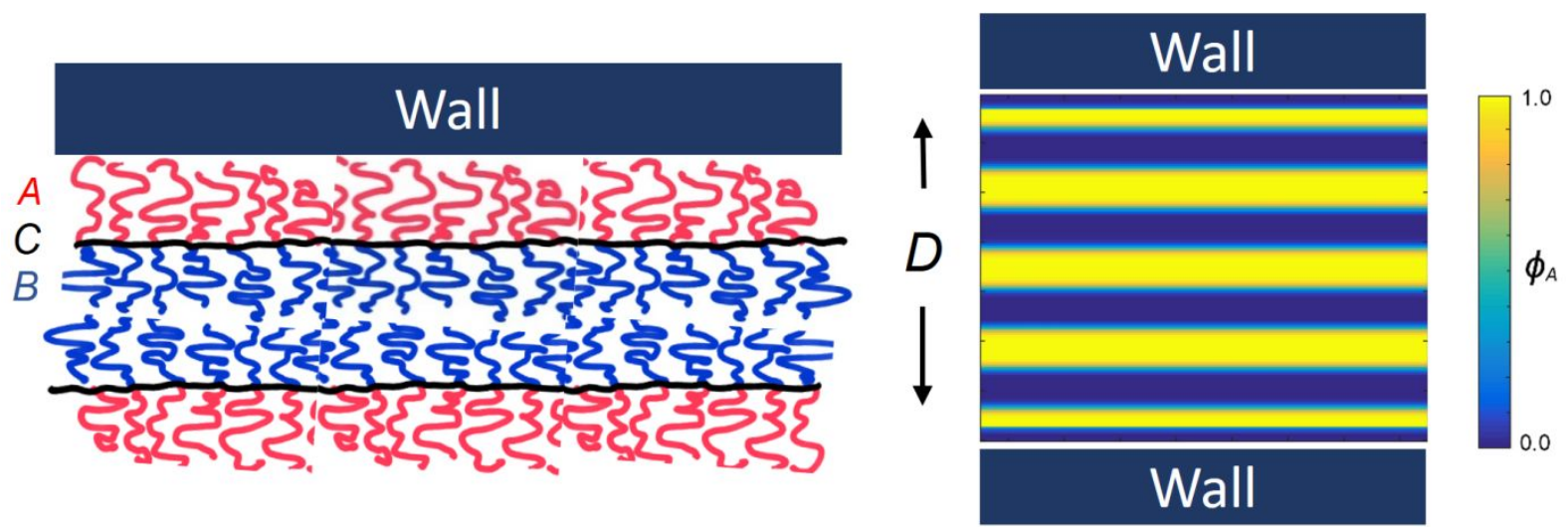

Figure S4. BBCPs self assembly near a wall. The extended worm-like backbone show preferentiality to lie parallel to the flat wall creating ordered domains parallel to wall as reflected in the density profile extracted from SCFT calculations.
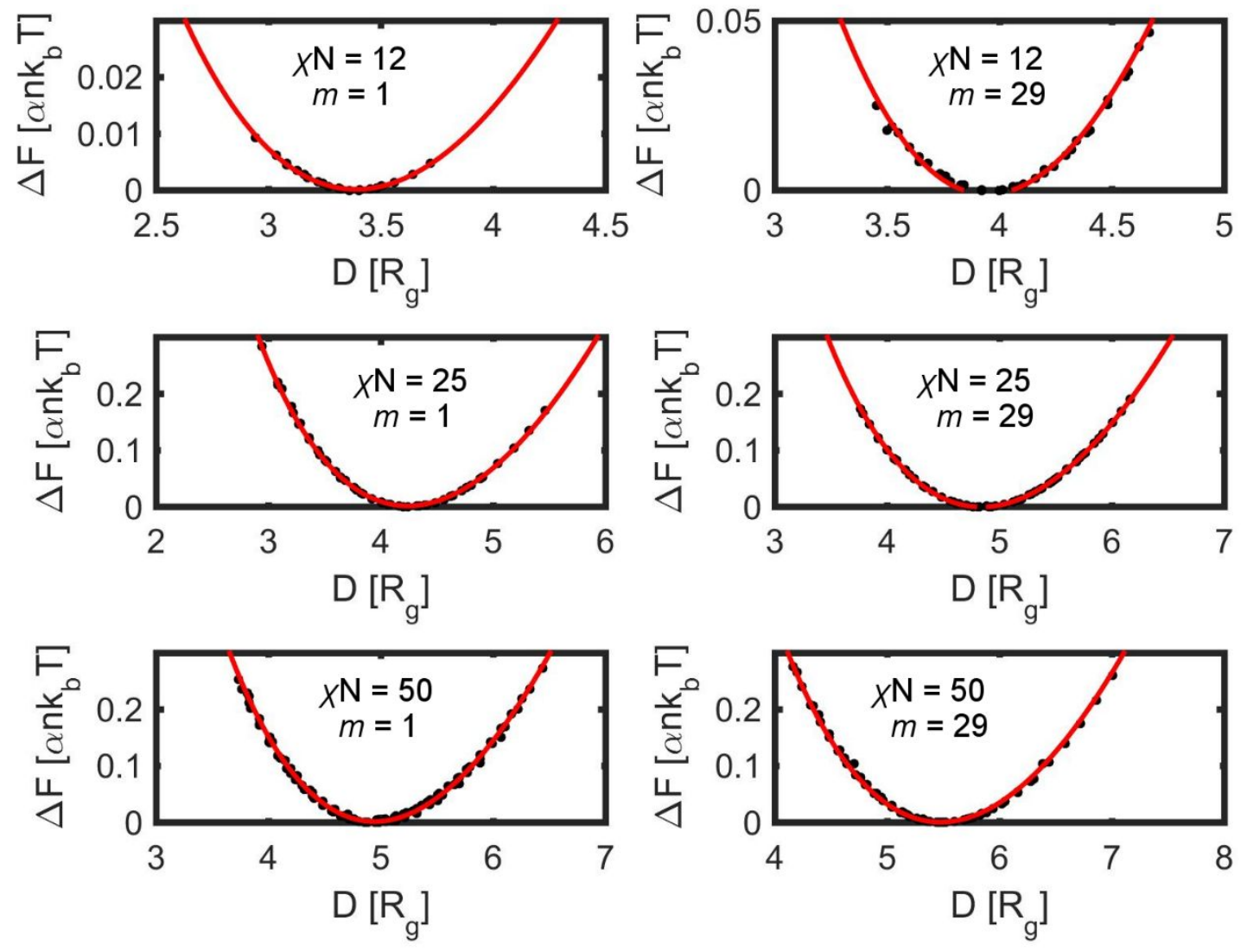

Figure S5. Master free energy showing the effect of trench spacing in real units $\left[R_{g}\right]$ on polymer strain energy. 